\title{
THE DIMENSION OF THE HILBERT SCHEME OF SPECIAL THREEFOLDS
}

\author{
GIAN MARIO BESANA AND MARIA LUCIA FANIA
}

\begin{abstract}
The Hilbert scheme of 3 -folds in $\mathbb{P}^{n}, n \geq 6$, that are scrolls over $\mathbb{P}^{2}$ or over a smooth quadric surface $\mathbf{Q} \subset \mathbb{P}^{3}$ or that are quadric or cubic fibrations over $\mathbb{P}^{1}$ is studied. All known such threefolds of degree $7 \leq d \leq 11$ are shown to correspond to smooth points of an irreducible component of their Hilbert scheme, whose dimension is computed.
\end{abstract}

\section{INTRODUCTION}

The classification of complex projective manifolds of low degree, extending the classical works of Weil, [32, and Swinnerton Dyer, 31, has been conducted in the recent past as a three-step process. Maximal lists of possible manifolds are first compiled, according to the admissible values of their numerical invariants. The second step deals with establishing the actual existence of manifolds in the lists, looking for effective constructions of explicit examples. Finally, the Hilbert scheme of existing manifolds with given Hilbert polynomial is investigated.

The first step of the program has seen over the years the successful work of several authors. Manifolds of degree up to eight have been classified by Ionescu, 20, 22, 21], Okonek, 25], 26], Abo, Decker and Sasakura, 1]. Other authors have considered the special case of manifolds of fixed low codimension. Beltrametti, Schneider and Sommese, 2, 3, Ottaviani, [29], Braun, Ottaviani, Schneider and Schreyer, 8], classified 3-folds in $\mathbb{P}^{5}$ of degree up to twelve, and recently Bertolini, [5], classified 3-folds of degree twelve in $\mathbb{P}^{6}$. The classification of manifolds of dimension $n \geq 3$, regardless of the codimension, of degree nine and ten was accomplished by Fania and Livorni, 14, [15], while Besana and Biancofiore, 6], considered the case of degree eleven.

Many of the authors cited above also dealt with the issue of the actual existence of the classified manifolds, although open questions still remain

1991 Mathematics Subject Classification. Primary 14J30,14M07,14N25; Secondary 14 N30.

Partially supported by MIUR of the Italian Government in the framework of the National Research Project (Cofin 2002) Geometria sulle Varietà Algebriche.

The material in this paper is, in part, based upon work supported by the National Science Foundation (NSF) under Grant No. 0125068. Any opinions, findings and conclusions or recommendations expressed in this material are those of the authors and do not necessarily reflect the views of the NSF. 
in this arena. Further contributions to this second step of the program can also be found in works of Okonek, 27, and Biancofiore and Fania, 7].

As to the Hilbert schemes, general results are known only for fixed low codimension. In codimension two Ellingsrud, 12, considered arithmetically Cohen Macaulay varieties regardless of their degree, Ionescu dealt with such cases among the manifolds in 22, while the Hilbert scheme of a special class of 3 -folds in $\mathbb{P}^{5}$ was studied by Fania and Mezzetti, 16. General results in codimension two are also due to M.C. Chang, 9], 10].

In codimension 3, Kleppe and Miró-Roig, 24, computed the dimension of the open subset of the Hilbert scheme of arithmetically Gorenstein closed subschemes, independently of their degree, while Kleppe, Migliore, MiróRoig, Nagel and Peterson, 23], dealt with good determinantal subschemes.

In this paper, four classes of 3 -folds in $\mathbb{P}^{n}$ with $n \geq 6$ are considered. Precisely we deal with 3 -folds which are scrolls over $\mathbb{P}^{2}$ or over a smooth quadric surface $\mathbf{Q}$ and 3 -folds which are quadric or cubic fibrations over $\mathbb{P}^{1}$. Their geometric special structure is exploited in order to find mild conditions guaranteeing that these manifolds are unobstructed and their Hilbert scheme has an irreducible component which is smooth at the point corresponding to the given variety. Explicit formulas to compute the dimension of such components are given.

All manifolds in the above classes, of degree $7 \leq d \leq 11$, and known to exist, are shown to be unobstructed. The dimension of an irreducible component of their Hilbert scheme is computed. In some cases, a dense open subset of such an irreducible component of the Hilbert scheme is shown to be the locus of good determinantal subschemes with the given Hilbert polynomial (see Section 5 for definitions).

The paper is structured as follows: in Section 2 we collect all the necessary notation and background material, including the fundamental theorem of Grothendieck on the existence of the Hilbert scheme; in Section 3 unobstruction results for scrolls over $\mathbb{P}^{2}$ and $\mathbf{Q}$ are presented; Section 4 is devoted to the same results for quadric and cubic fibrations over $\mathbb{P}^{1}$; Section 5 explores the connections with results on unobstructed good determinantal subschemes presented in 23 .

\section{Notation And Preliminaries}

In this section notation is fixed and definitions and results which will be used throughout the paper are recalled.

Let $X$ be a complex projective manifold of dimension 3,3 -fold for short, and let $L$ be a very ample line bundle on $X$. Projective properties of $(X, L)$ are always referred to the embedding $X \subset \mathbb{P}^{n}$ given by the complete linear system associated with $L$. Therefore our 3 -folds are always linearly normal. We denote by $S$ and $C$, respectively, a smooth surface and curve, obtained as transverse intersection of $X$ with respectively 1 and 2 general elements of $|L|$. For any coherent sheaf $\mathcal{F}$ on $X, h^{i}(X, \mathcal{F})$ is the complex dimension of 
$H^{i}(X, \mathcal{F})$. When the ambient variety is understood, we often write $H^{i}(\mathcal{F})$ and $h^{i}(\mathcal{F})$ respectively for $H^{i}(X, \mathcal{F})$ and $h^{i}(X, \mathcal{F})$. The following notation will be used throughout this work.

$\chi(L)=\sum(-1)^{i} h^{i}(L)$, the Euler characteristic of $L$;

$L_{\left.\right|_{Y}}$ the restriction of $L$ to a subvariety $Y$;

$K_{X}$ the canonical bundle of $X$. When the context is clear, $X$ may be dropped;

$q(S)=h^{1}\left(\mathcal{O}_{S}\right)$, the irregularity of $S$;

$p_{g}(S)=h^{0}\left(K_{S}\right)$, the geometric genus of $S$;

$c_{i}=c_{i}(X)$, the $i^{\text {th }}$ Chern class of $X$;

$d=\operatorname{deg} X=L^{3}$, the degree of $X$ in the embedding given by $L$;

$g=g(X)$, the sectional genus of $(X, L)$ defined by $2 g-2=(K+$ $2 L) L^{2}$

Q, a smooth quadric hypersurface embedded in $\mathbb{P}^{3}$;

$\lceil x\rceil$, the ceiling of a real number $x$, i.e. the smallest integer greater than, or equal to, $x$.

Cartier divisors, their associated line bundles and the invertible sheaves of their holomorphic sections are used with no distinction. Mostly additive notation is used for their group. Multiplicative notation (juxtaposition) will be used for intersection of cycles and Chern classes.

Definition 2.1. A pair $(X, L)$, where $L$ is an ample line bundle on a 3fold $X$, is a scroll, or a hyperquadric fibration, or a Del Pezzo fibration over a normal variety $Y$ if there exist an ample line bundle $M$ on $Y$ and a surjective morphism $\varphi: X \rightarrow Y$ with connected fibers such that $K_{X}+$ $(4-\operatorname{dim} Y) L=\varphi^{*}(M)$ or, respectively, $K_{X}+(3-\operatorname{dim} Y) L=\varphi^{*}(M)$ or $K_{X}+(2-\operatorname{dim} Y) L=\varphi^{*}(M)$.

Remark 2.2. Let $(X, L)$ be a scroll over a surface $Y$. Then, see 4 14.1.3, $X \cong \mathbb{P}(\mathcal{E})$, where $\mathcal{E}=\varphi_{*}(L)$ and $L$ is the tautological line bundle on $\mathbb{P}(\mathcal{E})$. It is known, see for example 4 . Section 11.1, that $S$ is the blow up of $Y$ at $c_{2}(\mathcal{E})$ points, $\chi\left(\mathcal{O}_{Y}\right)=\chi\left(\mathcal{O}_{S}\right)$ and $d=c_{1}^{2}(\mathcal{E})-c_{2}(\mathcal{E})$.

Remark 2.3. Let $(X, L)$ be a 3-dimensional manifold which is either a hyperquadric fibration or a Del Pezzo fibration of fiber degree 3 over $\mathbb{P}^{1}$, $\varphi: X \rightarrow \mathbb{P}^{1}$, which is embedded by $|L|$ in $\mathbb{P}^{n}$. Let $F \in\left|\varphi^{*}\left(\mathcal{O}_{\mathbb{P}^{1}}(1)\right)\right|$ be a fiber of $\varphi$, then $L^{2} F=\alpha=2,3$, respectively. As $L$ is very ample, 20, 17] and [11] show that there is a rank 4 vector bundle over $\mathbb{P}^{1}, \mathcal{E}=\varphi_{*}(L)=$ $\mathcal{O}_{\mathbb{P}^{1}}\left(a_{4}\right) \oplus \cdots \oplus \mathcal{O}_{\mathbb{P}^{1}}\left(a_{1}\right)$ and an embedding $\iota: X \rightarrow \mathbb{P}(\mathcal{E})$, such that $L=$ $\iota^{*}\left(\mathcal{O}_{\mathbb{P}(\mathcal{E})}(1)\right)$. Note that $X$ is a divisor on $\mathbb{P}(\mathcal{E})$ and $X \in\left|\mathcal{O}_{\mathbb{P}(\mathcal{E})}(\alpha)+\rho^{*} \mathcal{O}_{\mathbb{P}^{1}}(b)\right|$ where $\rho: \mathbb{P}(\mathcal{E}) \rightarrow \mathbb{P}^{1}$ is the projection map. Let $e=\operatorname{deg} \mathcal{E}$, then

$$
\left\{\begin{array} { l } 
{ d = \alpha e + b } \\
{ 2 g - 2 = \alpha ( e + b - 2 ) + ( \alpha - 2 ) d }
\end{array} \quad \text { or } \quad \left\{\begin{array}{l}
b=\frac{d-d \alpha+2 g-2+2 \alpha}{\alpha-1} \\
e=-\frac{2(g-1+\alpha-d \alpha+d)}{\alpha(\alpha-1)}
\end{array}\right.\right.
$$


2.1. The Hilbert Scheme. The existence of the Hilbert scheme for closed subschemes of $\mathbb{P}^{n}$ with given Hilbert polynomial was established by Grothendieck, [18. The following formulation of his basic result is due to Sommese, 30.

Proposition 2.4 ([18, 30]). Let $Z$ be a smooth connected projective variety. Let $X$ be a connected submanifold of $Z$ with $H^{1}(X, N)=0$ where $N$ is the normal bundle of $X$. Then there exist irreducible projective varieties $\mathcal{Y}$ and $\mathcal{H}$ with the following properties:

(i) $\mathcal{Y} \subset \mathcal{H} \times Z$ and the map $p: \mathcal{Y} \rightarrow \mathcal{H}$ induced by the product projection is a flat surjection,

(ii) there is a smooth point $x \in \mathcal{H}$ with $p$ of maximal rank in a neighborhood of $p^{-1}(x)$,

(iii) $q$ identifies $p^{-1}(x)$ with $X$ where $q: \mathcal{Y} \rightarrow Z$ is the map induced by the product projection, and

(iv) $H^{0}(N)$ is naturally identified with $T_{\mathcal{H}, x}$ where $T_{\mathcal{H}, x}$ is the Zariski tangent space of $\mathcal{H}$ at $x$.

\section{Hilbert scheme of 3 -Dimensional scrolls over $\mathbb{P}^{2}$ OR $\mathbf{Q}$}

Let $(X, L)=\left(\mathbb{P}(\mathcal{E}), \mathcal{O}_{\mathbb{P}(\mathcal{E})}(1)\right)$ be a smooth 3 -fold of sectional genus $g$ and degree $d$ which is a scroll over $\mathbb{P}^{2}$, as in Definition 2.1 and Remark 2.2 Let $X$ be embedded by $|L|$ in $\mathbb{P}^{n}$. The following proposition shows that, under mild conditions on the embedding and on the splitting type of $\mathcal{E}, X$ is unobstructed.

Proposition 3.1. Let $(X, L)=\left(\mathbb{P}(\mathcal{E}), \mathcal{O}_{\mathbb{P}(\mathcal{E})}(1)\right)$ be a 3-dimensional scroll over $\mathbb{P}^{2}$ of degree $d$ and sectional genus $g$. Let $X$ be embedded by $|L|$ in $\mathbb{P}^{n}$. Let $c_{1}(\mathcal{E})=\mathcal{O}_{\mathbb{P}^{2}}\left(e_{1}\right), e_{2}=c_{2}(\mathcal{E})$. Assume:

i) $H^{1}(X, L)=0$;

ii) there exists a line $\ell \subset \mathbb{P}^{2}$ such that $\mathcal{E}_{\left.\right|_{\ell}}=\mathcal{O}_{\mathbb{P}^{1}}(a) \oplus \mathcal{O}_{\mathbb{P}^{1}}\left(e_{1}-a\right)$ where $\frac{e_{1}}{2} \leq a \leq \frac{e_{1}}{2}+1$.

Then the Hilbert scheme of $X$ has an irreducible component, $\mathcal{H}$, which is smooth at the point representing $X$ and

$$
\operatorname{dim} \mathcal{H}=(d+2)(n-3)+\frac{3 e_{1}}{2}(n+1)-\frac{e_{1}^{2}}{2}(n-5)-4
$$

Proof. Let $N$ denote the normal bundle of $X$ in $\mathbb{P}^{n}$. The statement will follow from Proposition 2.4 by showing that $H^{1}(X, N)=0$ and conducting an explicit computation of $h^{0}(X, N)$. Let

$$
0 \longrightarrow \mathcal{O}_{X} \longrightarrow \mathcal{O}_{X}(1)^{\oplus(n+1)} \longrightarrow T_{\mathbb{P}^{n} \mid X} \longrightarrow 0
$$

be the Euler sequence on $\mathbb{P}^{n}$ restricted to $X$. As $(X, L)$ is a scroll over $\mathbb{P}^{2}$,

$$
H^{i}\left(X, \mathcal{O}_{X}\right)=H^{i}\left(\mathbb{P}^{2}, \mathcal{O}_{\mathbb{P}^{2}}\right)=0, \quad \text { for } \quad i \geq 1
$$


Let $S \in|L|$ and $C \in\left|L_{S}\right|$ be a general surface and curve section. From their structure sequences, noting that $S$ is rational, it follows that

$$
H^{2}(X, L)=H^{3}(X, L)=0 \text {. }
$$

Because $H^{1}(X, L)=0$ by assumption, from (3), (4) and the cohomology sequence associated to (2) it follows that $H^{i}\left(X, T_{\mathbb{P}^{n} \mid X}\right)=0$ for $i \geq 1$. Therefore the exact sequence

$$
0 \longrightarrow T_{X} \longrightarrow T_{\mathbb{P}^{n} \mid X} \longrightarrow N \longrightarrow 0
$$

gives

$$
H^{i}(X, N)=H^{i+1}\left(X, T_{X}\right) \quad \text { for } \quad i \geq 1 .
$$

In particular $H^{3}(X, N)=0$ for dimension reasons. To compute $H^{j}\left(X, T_{X}\right), j=$ 2,3 , let $\varphi: \mathbb{P}(\mathcal{E}) \longrightarrow \mathbb{P}^{2}$ be the scroll map, and consider the relative cotangent bundle sequence:

$$
0 \rightarrow \varphi^{*}\left(\Omega_{\mathbb{P}^{2}}^{1}\right) \rightarrow \Omega_{X}^{1} \rightarrow \Omega_{X \mid \mathbb{P}^{2}}^{1} \longrightarrow 0 .
$$

From (7) and the Whitney sum one obtains

$$
c_{1}\left(\Omega_{X}^{1}\right)=c_{1}\left(\varphi^{*}\left(\Omega_{\mathbb{P}^{2}}^{1}\right)\right)+c_{1}\left(\Omega_{X \mid \mathbb{P}^{2}}^{1}\right)
$$

and thus

$$
\Omega_{X \mid \mathbb{P}^{2}}^{1}=K_{X}+\varphi^{*}\left(\mathcal{O}_{\mathbb{P}^{2}}(3)\right) .
$$

The adjunction theoretic characterization of the scroll then gives

$$
\Omega_{X \mid \mathbb{P}^{2}}^{1}=K_{X}+\varphi^{*}\left(\mathcal{O}_{\mathbb{P}^{2}}(3)\right)=-2 L+\varphi^{*}\left(\mathcal{O}_{\mathbb{P}^{2}}\left(e_{1}\right)\right)
$$

that, combined with the dual of (7), gives

$$
0 \rightarrow 2 L-\varphi^{*}\left(\mathcal{O}_{\mathbb{P}^{2}}\left(e_{1}\right)\right) \rightarrow T_{X} \rightarrow \varphi^{*}\left(T_{\mathbb{P}^{2}}\right) \rightarrow 0 .
$$

As the cohomology of $\varphi^{*}\left(T_{\mathbb{P}^{2}}\right)$ is easily computed, we devote our attention to the cohomology of $2 L-\varphi^{*}\left(\mathcal{O}_{\mathbb{P}^{2}}\left(e_{1}\right)\right)$. Noticing that $R^{i} \varphi_{*}(2 L)=0$ for $i \geq 1$ (see [19, pg 253), projection formula and Leray's spectral sequence give

$$
H^{i}\left(X, 2 L-\varphi^{*}\left(\mathcal{O}_{\mathbb{P}^{2}}\left(e_{1}\right)\right)\right) \cong H^{i}\left(\mathbb{P}^{2}, S^{2} \mathcal{E} \otimes \mathcal{O}_{\mathbb{P}^{2}}\left(-e_{1}\right)\right) .
$$

Therefore

$$
H^{3}\left(X, 2 L-\varphi^{*}\left(\mathcal{O}_{\mathbb{P}^{2}}\left(e_{1}\right)\right)\right)=0
$$

for dimension reasons.

Let $\ell$ be a line in $\mathbb{P}^{2}$ as in assumption ii).

It is

$$
(\mathcal{E} \otimes \mathcal{E})_{\mid \ell}=\mathcal{O}_{\mathbb{P}^{1}}(2 a) \oplus \mathcal{O}_{\mathbb{P}^{1}}\left(2\left(e_{1}-a\right)\right) \oplus \mathcal{O}_{\mathbb{P}^{1}}\left(e_{1}\right) \oplus \mathcal{O}_{\mathbb{P}^{1}}\left(e_{1}\right) .
$$

As $\operatorname{det}(\mathcal{E})=\mathcal{O}_{\mathbb{P}^{2}}\left(e_{1}\right)$ and $\mathcal{E} \otimes \mathcal{E}=\operatorname{det}(\mathcal{E}) \oplus S^{2} \mathcal{E}$ it follows that

$$
S^{2} \mathcal{E}_{\mid \ell}=\mathcal{O}_{\mathbb{P}^{1}}(2 a) \oplus \mathcal{O}_{\mathbb{P}^{1}}\left(2\left(e_{1}-a\right)\right) \oplus \mathcal{O}_{\mathbb{P}^{1}}\left(e_{1}\right) \text {. }
$$


Tensoring the structure sequence of $\ell$ on $\mathbb{P}^{2}$ with $S^{2} \mathcal{E} \otimes \mathcal{O}_{\mathbb{P}^{2}}(t)$ gives

$$
0 \rightarrow S^{2} \mathcal{E}(t-1) \longrightarrow S^{2} \mathcal{E}(t) \rightarrow\left(S^{2} \mathcal{E} \otimes \mathcal{O}_{\mathbb{P}^{2}}(t)\right)_{\left.\right|_{\ell}} \rightarrow 0
$$

where

$$
\left(S^{2} \mathcal{E} \otimes \mathcal{O}_{\mathbb{P}^{2}}(t)\right)_{\left.\right|_{\ell}}=\mathcal{O}_{\mathbb{P}^{1}}(2 a+t) \oplus \mathcal{O}_{\mathbb{P}^{1}}\left(2\left(e_{1}-a\right)+t\right) \oplus \mathcal{O}_{\mathbb{P}^{1}}\left(e_{1}+t\right) .
$$

Because $a \geq \frac{e_{1}}{2}$ one can see that the minimum of the integers $2 a, 2\left(e_{1}-a\right), e_{1}$ is $2\left(e_{1}-a\right)$.

The cohomology sequence associated with (11) then gives

$$
H^{2}\left(S^{2} \mathcal{E}(t-1)\right)=H^{2}\left(S^{2} \mathcal{E}(t)\right) \quad \forall t \geq 2\left(a-e_{1}\right)-1 .
$$

Thus

$H^{2}\left(S^{2} \mathcal{E}\left(2\left(a-e_{1}\right)-2\right)\right)=H^{2}\left(S^{2} \mathcal{E}\left(2\left(a-e_{1}\right)-1\right)\right)=\cdots=H^{2}\left(S^{2} \mathcal{E}(s)\right)=\ldots$

Therefore Serre's vanishing theorem gives

$$
H^{2}\left(S^{2} \mathcal{E}(s)\right)=0, \quad \text { for all } s \geq 2\left(a-e_{1}\right)-2 .
$$

In particular $H^{2}\left(S^{2} \mathcal{E}\left(-e_{1}\right)\right)=0$ because of our assumption $a \leq \frac{e_{1}}{2}+1$, and thus, from the cohomology sequence associated to (8), (9), and dimension reasons, it follows that $H^{2}\left(X, T_{X}\right)=H^{2}\left(\mathbb{P}^{2}, f^{*} T_{\mathbb{P}^{2}}\right)$ and $H^{3}\left(X, T_{X}\right)=0$. On the other hand, by Leray spectral sequence,

$$
H^{2}\left(\mathbb{P}^{2}, \varphi^{*} T_{\mathbb{P}^{2}}\right)=H^{2}\left(\mathbb{P}^{2}, T_{\mathbb{P}^{2}}\right)=0 .
$$

Hence $H^{i}\left(X, T_{X}\right)=0, i=2,3$ and thus, by (6),

$$
H^{i}(X, N)=0 \quad i=1,2,3 .
$$

According to Proposition 2.4 there exists an irreducible component $\mathcal{H}$ of the Hilbert scheme of $X \subset \mathbb{P}^{n}$. The dimension of $\mathcal{H}$, by Proposition 2.4 (iv), will be given by $h^{0}(X, N)=\chi(N)$. The Hirzebruch-Riemann-Roch theorem gives

$$
\begin{aligned}
\chi(N)= & \frac{1}{6}\left(n_{1}^{3}-3 n_{1} n_{2}+3 n_{3}\right)+\frac{1}{4} c_{1}\left(n_{1}^{2}-2 n_{2}\right)+\frac{1}{12}\left(c_{1}^{2}+c_{2}\right) n_{1} \\
& +(n-3) \chi\left(\mathcal{O}_{X}\right)
\end{aligned}
$$

where $n_{i}=c_{i}(N)$, and $c_{i}=c_{i}(X)$.

Chern classes of $N$ can be obtained from (15):

$$
\begin{aligned}
n_{1}= & K+(n+1) L \\
n_{2}= & \frac{1}{2} n(n+1) L^{2}+(n+1) L K+K^{2}-c_{2} ; \\
n_{3}= & \frac{1}{6}(n-1) n(n+1) L^{3}+\frac{1}{2} n(n+1) K L^{2}+(n+1) K^{2} L-(n+1) c_{2} L \\
& -2 c_{2} K+K^{3}-c_{3} .
\end{aligned}
$$


The numerical invariants of $X$ can be easily computed:

$$
\begin{aligned}
K L^{2} & =-2 d+e_{1}^{2}-3 e_{1} ; & K^{2} L & =4 d-3 e_{1}^{2}+6 e_{1}+9 ; \\
c_{2} L & =3 e_{1}+3 ; & K^{3} & =-8 d+6 e_{1}^{2}-54 ; \\
-K c_{2} & =24 ; & c_{3} & =6 .
\end{aligned}
$$

Plugging these in (14) and the results in (13) one gets

$$
\chi(N)=-10+\frac{3}{2} e_{1}-3 d+\frac{5}{2} e_{1}^{2}+2 n+\frac{3}{2} n e_{1}+d n-\frac{1}{2} n e_{1}^{2} .
$$

Let $(X, L)=\left(\mathbb{P}(\mathcal{E}), \mathcal{O}_{\mathbb{P}(\mathcal{E})}(1)\right)$ be a smooth 3 -fold of sectional genus $g$ and degree $d$ which is a scroll over a smooth quadric surface $\mathbf{Q} \subset \mathbb{P}^{3}$, as in Definition 2.1 and Remark 2.2. Let $X$ be embedded by $|L|$ in $\mathbb{P}^{n}$. The following proposition shows that, under mild conditions on the embedding and on the splitting type of $\mathcal{E}, X$ is unobstructed.

Proposition 3.2. Let $(X, L)=\left(\mathbb{P}(\mathcal{E}), \mathcal{O}_{\mathbb{P}(\mathcal{E})}(1)\right)$ be a 3-dimensional scroll over $\mathbf{Q}$ of degree $d$ and sectional genus $g$. Let $X$ be embedded by $|L|$ in $\mathbb{P}^{n}$ and let $c_{1}(\mathcal{E})=\mathcal{O}_{\mathbf{Q}}\left(e_{11}, e_{12}\right), c_{2}(\mathcal{E})=e_{2}$. Assume:

i) $H^{1}(X, L)=0$;

ii) there exists a line $\ell_{1} \in|\mathcal{O}(1,0)|$ and a line $\ell_{2} \in|\mathcal{O}(0,1)|$ such that:

$$
\begin{aligned}
& \mathcal{E}_{\left.\right|_{\ell_{1}}}=\mathcal{O}_{\mathbb{P}^{1}}\left(\left\lceil\frac{e_{12}}{2}\right\rceil\right) \oplus \mathcal{O}_{\mathbb{P}^{1}}\left(e_{12}-\left\lceil\frac{e_{12}}{2}\right\rceil\right), \\
& \mathcal{E}_{\left.\right|_{\ell_{2}}}=\mathcal{O}_{\mathbb{P}^{1}}\left(\left\lceil\frac{e_{11}}{2}\right\rceil\right) \oplus \mathcal{O}_{\mathbb{P}^{1}}\left(e_{11}-\left\lceil\frac{e_{11}}{2}\right\rceil\right) .
\end{aligned}
$$

Then the Hilbert scheme of $X$ has an irreducible component, $\mathcal{H}$, which is smooth at the point representing $X$ and

$$
\operatorname{dim} \mathcal{H}=(d+2)(n-3)+\left(e_{11}+e_{12}\right)(n+1)-e_{11} e_{12}(n-5)-2 .
$$

Proof. The proof proceeds exactly as in Proposition 3.1. Letting $N$ denote the normal bundle of $X$ in $\mathbb{P}^{n}$, the computation of $H^{i}(N)$ relies this time on $H^{i}\left(\mathbf{Q}, S^{2} \mathcal{E} \otimes \mathcal{O}_{\mathbf{Q}}\left(-e_{11},-e_{12}\right)\right)$.

Let $\ell_{1}$ and $\ell_{2}$ be lines in $\left|\mathcal{O}_{\mathbf{Q}}(1,0)\right|$ and $\left|\mathcal{O}_{\mathbf{Q}}(0,1)\right|$, respectively.

We can assume that $\mathcal{E}_{\left.\right|_{\ell_{1}}}=\mathcal{O}_{\ell_{1}}(a) \oplus \mathcal{O}_{\ell_{1}}\left(e_{12}-a\right)$ and $\mathcal{E}_{\left.\right|_{\ell_{2}}}=\mathcal{O}_{\ell_{2}}(b) \oplus$ $\mathcal{O}_{\ell_{2}}\left(e_{11}-b\right)$ with $a \geq \frac{e_{12}}{2}$ and $b \geq \frac{e_{11}}{2}$.

Tensoring the structure sequences of $\ell_{1}$ and $\ell_{2}$ on $\mathbf{Q}$ by $S^{2} \mathcal{E}(t-e, t-1)$ and $S^{2} \mathcal{E}(t-e-1, t)$ respectively, where $e=e_{11}-e_{12}$, we get:

$$
\begin{gathered}
0 \rightarrow S^{2} \mathcal{E}(t-e-1, t-1) \rightarrow S^{2} \mathcal{E}(t-e, t-1) \rightarrow \\
\mathcal{O}_{\ell_{1}}(2 a+t-1) \oplus \mathcal{O}_{\ell_{1}}\left(e_{12}+t-1\right) \oplus \mathcal{O}_{\ell_{1}}\left(2\left(e_{12}-a\right)+t-1\right) \rightarrow 0,
\end{gathered}
$$

$$
\begin{aligned}
& \quad 0 \rightarrow S^{2} \mathcal{E}(t-e-1, t-1) \rightarrow S^{2} \mathcal{E}(t-e-1, t) \rightarrow \\
& \mathcal{O}_{\ell_{2}}(2 b+t-e-1) \oplus \mathcal{O}_{\ell_{2}}\left(e_{11}+t-e-1\right) \oplus \mathcal{O}_{\ell_{2}}\left(2\left(e_{11}-b\right)+t-e-1\right) \rightarrow 0 .
\end{aligned}
$$


Assume

$$
2\left(e_{12}-a\right)+t-1 \geq-1,
$$

that is $t \geq 2\left(a-e_{12}\right)=2\left(a-e_{11}+e\right)$, and assume

$$
2\left(e_{11}-b\right)+t-e-1 \geq-1,
$$

that is $t \geq 2\left(b-e_{11}\right)+e$. Then the cohomology sequences associated with (15) and (16) give

$$
H^{2}\left(S^{2} \mathcal{E}(t-e, t-1)\right)=H^{2}\left(S^{2} \mathcal{E}(t-e-1, t-1)\right)=H^{2}\left(S^{2} \mathcal{E}(t-e-1, t)\right) .
$$

Tensoring now the structure sequence of $\ell_{1}$ with $S^{2} \mathcal{E}(t-e, t)$ one gets:

$$
\begin{array}{r}
0 \longrightarrow S^{2} \mathcal{E}(t-e-1, t) \longrightarrow S^{2} \mathcal{E}(t-e, t) \longrightarrow \mathcal{O}_{\ell_{1}}(2 a+t) \oplus \\
\oplus \mathcal{O}_{\ell_{1}}\left(e_{12}+t\right) \oplus \mathcal{O}_{\ell_{1}}\left(2\left(e_{12}-a\right)+t\right) \longrightarrow 0
\end{array}
$$

From (17) it follows:

$$
H^{2}\left(S^{2} \mathcal{E}(t-e, t)\right)=H^{2}\left(S^{2} \mathcal{E}(t-e-1, t)\right) .
$$

Thus, under assumptions (17) and (18), from (19) and (21), it follows that

$$
H^{2}\left(S^{2} \mathcal{E}(t-e, t)\right)=H^{2}\left(S^{2} \mathcal{E}(t-e-1, t-1)\right),
$$

thus by Serre's vanishing theorem, under the same assumptions,

$$
H^{2}\left(S^{2} \mathcal{E}(t-e-1, t-1)\right)=0 .
$$

To obtain the desired vanishing $H^{2}\left(S^{2} \mathcal{E}\left(-e_{11},-e_{12}\right)\right)=0$ the following conditions need to be satisfied:

(a) $2\left(a-e_{12}\right)-e-1 \leq-e_{11}$;

(b) $2\left(b-e_{11}\right)+e-1 \leq-e_{12}$.

Condition (a) along with the fact that $a \geq \frac{e_{12}}{2}$ gives $a=\left\lceil\frac{e_{12}}{2}\right\rceil$. Analogously, condition (b) gives $b=\left\lceil\frac{e_{11}}{2}\right\rceil$.

Similarly to the proof of Proposition 3.1 we get $H^{i}\left(X, T_{X}\right)=0$ for $i=2,3$, thus by (6)

$$
H^{i}(X, N)=0 \quad i=1,2,3,
$$

and therefore, by Proposition 2.4 we have established the existence of an irreducible component $\mathcal{H}$ of the Hilbert scheme of $X \subset \mathbb{P}^{n}$.

The dimension of $\mathcal{H}$, as in Proposition [3.1] is obtained via HirzebruchRiemann-Roch theorem, using (5D) to compute the Chern classes of $N$.

Let us now consider the numerical invariants of $X$. Let $H_{i}=\varphi^{*}\left(\ell_{i}\right)$ and let $F$ denote a fiber of $\varphi$. One can easily obtain the following relations in the cohomology ring of $X=\mathbb{P}(\mathcal{E})$ :

$$
\begin{aligned}
& L^{3}=d ; \quad L^{2} H_{1}=e_{12} ; \quad L^{2} H_{2}=e_{11} ; \quad H_{1}^{3}=H_{2}^{3}=0 ; \\
& L^{2} F=1 ; \quad L H_{1} H_{2}=1 ; \quad H_{1} F=H_{2} F=0 ; \quad L H_{1}^{2}=L H_{2}^{2}=0 .
\end{aligned}
$$


Using (22) we get:

$$
\begin{array}{rlrl}
K L^{2} & =-2 d+2\left(e_{11} e_{12}-e_{11}-e_{12}\right) ; & c_{2} L & =2\left(e_{11}+e_{12}\right)+4 ; \\
K^{2} L & =4 d+4\left(e_{11}+e_{12}\right)-6 e_{11} e_{12}+8 ; & K^{3} & =-8 d+12 e_{11} e_{12}-48 ; \\
-K c_{2} & =24
\end{array}
$$

and thus, recalling that $c_{3}=8$,

$h^{0}(X, N)=\chi(N)=n\left(d+2+e_{11}+e_{12}-e_{11} e_{12}\right)-8-3 d+e_{11}+e_{12}+5 e_{11} e_{12}$.

As a Corollary to Proposition 3.1 we show that all the known 3 -folds scrolls over $\mathbb{P}^{2}$ of degree $7 \leq d \leq 12$ are unobstructed and we compute the dimension of the irreducible component of the Hilbert scheme to which they belong. Note that no such varieties exist for $d \leq 6$.

Corollary 3.3. Let $(X, L)=\left(\mathbb{P}(\mathcal{E}), \mathcal{O}_{\mathbb{P}(\mathcal{E})}(1)\right)$ be a 3-dimensional scroll over $\mathbb{P}^{2}$. Let $X$ be embedded by $|L|$ in $\mathbb{P}^{n}$, with degree $d$ and sectional genus $g$ as in the table below. Then the Hilbert scheme of $X \subset \mathbb{P}^{n}$ has an irreducible component, $\mathcal{H}$, which is smooth at the point representing $X$ and of dimension as in the rightmost column of the table.

\begin{tabular}{|c|c|c|c|c|c|c|}
\hline$d$ & $g$ & $n$ & $c_{1}(\mathcal{E})$ & $c_{2}(\mathcal{E})$ & Reference & $\operatorname{dim} \mathcal{H}$ \\
\hline \hline 7 & 3 & 6 & 4 & 9 & {$[22]$ Prop. 1.3 } & 57 \\
\hline 8 & 3 & 7 & 4 & 8 & {$[22]$ Prop. 1.3 } & 68 \\
\hline 9 & 3 & 8 & 4 & 7 & {$[22]$ Prop. 1.3, } & 81 \\
& & & & {$[14]$ Prop. 3.1 and Remark 3.2 } & \\
\hline 10 & 3 & 9 & 4 & 6 & {$[22]$ Prop. 1.3, } & 96 \\
& & & & {$[15]$ Prop. 3.4 } & \\
\hline 10 & 6 & 6 & 5 & 15 & {$[15]$ Remark 5.3 } & 72 \\
\hline 12 & 3 & 11 & 4 & 4 & {$[20]$ Prop. 4.7 } & 132 \\
\hline
\end{tabular}

Proof. We will show that for all the cases in the above table, the hypothesis of Proposition 3.1 are satisfied. The structure sequences of a general surface section $S$ and curve section $C$ tensored with $L$ and $L_{\left.\right|_{S}}$ respectively, give

$$
h^{0}(L)-h^{1}(L)=3+d-g .
$$

On the other hand, $h^{0}(L)=n+1$ and thus

$$
h^{1}(L)=n-2-d+g .
$$

A simple check gives $h^{1}(L)=0$ for all the cases in the above table.

To establish the existence of a line $\ell$ as in hypothesis ii) of Proposition 3.1 we first consider the cases in the above table with $c_{1}(\mathcal{E})=4$. As $\mathcal{E}$ is ample, the generic splitting type of $\mathcal{E}$ is then either $\mathcal{O}_{\mathbb{P}^{1}}(2) \oplus \mathcal{O}_{\mathbb{P}^{1}}(2)$ or $\mathcal{O}_{\mathbb{P}^{1}}(3) \oplus \mathcal{O}_{\mathbb{P}^{1}}(1)$. In both cases, a generic line $\ell$ satisfies hypothesis ii).

In the case with $c_{1}(\mathcal{E})=5$ the possible splitting types are $\mathcal{O}_{\mathbb{P}^{1}}(3) \oplus \mathcal{O}_{\mathbb{P}^{1}}(2)$ or $\mathcal{O}_{\mathbb{P}^{1}}(4) \oplus \mathcal{O}_{\mathbb{P}^{1}}(1)$. If the generic splitting type is $\mathcal{O}_{\mathbb{P}^{1}}(3) \oplus \mathcal{O}_{\mathbb{P}^{1}}(2)$, then 
hypothesis ii) is satisfied for a generic line $\ell$. If the generic splitting type is $\mathcal{O}_{\mathbb{P}^{1}}(4) \oplus \mathcal{O}_{\mathbb{P}^{1}}(1)$, then $\mathcal{E}$ must be uniform. If not, there should exist a line on which $\mathcal{E}$ jumps, i.e. on which it splits as $\mathcal{O}_{\mathbb{P}^{1}}(a) \oplus \mathcal{O}_{\mathbb{P}^{1}}(b), a \geq b$, with $(4,1) \leq(a, b)$, lexicographically, see [28, p.29]; this is impossible as the only other admissible splitting type for $\mathcal{E}$ is $\mathcal{O}_{\mathbb{P}^{1}}(3) \oplus \mathcal{O}_{\mathbb{P}^{1}}(2)$, being $\mathcal{E}$ ample, and $(3,2) \leq(4,1)$, lexicographically.

Uniform 2-bundles on $\mathbb{P}^{2}$ either split or are of the form $T_{\mathbb{P}^{2}}(t)$, see 28 , Theorem 2.2.2, p. 211]. Both cases are ruled out as $c_{1}(\mathcal{E})=5$ and $c_{2}(\mathcal{E})=$ 15.

By Proposition 3.1 there exists an irreducible component $\mathcal{H}$ of the Hilbert scheme of $X \subset \mathbb{P}^{n}$ whose dimension can now be easily computed.

Remark 3.4. The 3 -dimensional scrolls over $\mathbb{P}^{2}$ of degree 11 whose invariants are the following:

\begin{tabular}{|c|c|c|c|c|c|c|}
\hline$d$ & $g$ & $n$ & $\overline{c_{1}(\mathcal{E})}$ & $\overline{c_{2}(\mathcal{E})}$ & Reference & $\operatorname{dim} \mathcal{H}$ \\
\hline 11 & 3 & 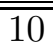 & 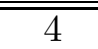 & $\overline{\overline{5}}$ & $\begin{array}{lll}6 & \text { Prop. } 4.2 .2\end{array}$ & $\overline{\overline{113}}$ \\
\hline 11 & 6 & 7 & 5 & 14 & $\begin{array}{lll}6 \text { 6 } & \text { Prop. } 5.2 .3\end{array}$ & $\overline{83}$ \\
\hline
\end{tabular}

also satisfy the hypothesis of Proposition 3.1. Unfortunately the existence of these scrolls is not known as yet.

As a Corollary to Proposition 3.2 we show that all the known 3-folds scrolls over $\mathbf{Q}$ of degree $8 \leq d \leq 11$ are unobstructed and we compute the dimension of the irreducible component of the Hilbert scheme to which they belong. Note that no such varieties exist for $d \leq 7$.

Corollary 3.5. Let $(X, L)=\left(\mathbb{P}(\mathcal{E}), \mathcal{O}_{\mathbb{P}(\mathcal{E})}(1)\right)$ be a 3-dimensional scroll over Q. Let $X$ be embedded by $|L|$ in $\mathbb{P}^{n}$, with degree $d$ and sectional genus $g$ as in the table below. Then the Hilbert scheme of $X \subset \mathbb{P}^{n}$ has an irreducible component, $\mathcal{H}$, which is smooth at the point representing $X$ and of dimension as in the rightmost column of the table.

\begin{tabular}{|c|c|c|c|c|c|c|}
\hline$d$ & $g$ & $n$ & $c_{1}(\mathcal{E})$ & $c_{2}(\mathcal{E})$ & Reference & $\operatorname{dim} \mathcal{H}$ \\
\hline \hline 8 & 4 & 6 & $\mathcal{O}_{\mathbf{Q}}(3,3)$ & 10 & {$[22]$ Prop. 1.6 } & 61 \\
\hline 9 & 4 & 7 & $\mathcal{O}_{\mathbf{Q}}(3,3)$ & 9 & {$[15]$ Remark 7.5 } & 72 \\
\hline 10 & 4 & 8 & $\mathcal{O}_{\mathbf{Q}}(3,3)$ & 8 & {$[15]$ Remark 3.5 } & 85 \\
\hline 11 & 4 & 9 & $\mathcal{O}_{\mathbf{Q}}(3,3)$ & 7 & $\underline{6}$ Remark 4.2.4 & 100 \\
\hline
\end{tabular}

Proof. We will show that for all the cases in the above table, the hypothesis of Proposition 3.2 are satisfied. To verify hypothesis i) of Proposition [3.2 one proceeds exactly as in the first part of the proof of Corollary 3.3 .

As $c_{1}(\mathcal{E})=\mathcal{O}_{\mathbf{Q}}(3,3)$, the splitting type of $\mathcal{E}$ on any line of both rulings is, $\mathcal{O}_{\mathbb{P}^{1}}(2) \oplus \mathcal{O}_{\mathbb{P}^{1}}(1)$, and thus hypothesis ii) is satisfied.

By Proposition 3.2 there exists an irreducible component $\mathcal{H}$ of the Hilbert scheme of $X \subset \mathbb{P}^{n}$ whose dimension can now be easily computed. 


\section{Hilbert scheme of 3 -Dimensional fibrations over $\mathbb{P}^{1}$ With Low FIBER DEGREE}

In this section we deal with 3 -folds that are fibrations over $\mathbb{P}^{1}$ with low fiber degree. As in the case of scrolls over surfaces we will see that under mild conditions these 3 -folds are also unobstructed. The dimension of the irreducible component of the Hilbert scheme to which they belong is computed.

Further notation is introduced here below.

4.1. Notation. Let $(X, L)$ be a 3-dimensional manifold which is either a hyperquadric fibration or a Del Pezzo fibration of fiber degre 3 over $\mathbb{P}^{1}$, as in Definiiton 2.1. Let $X$ be embedded by $|L|$ in $\mathbb{P}^{n}$. Let $F \in\left|\varphi^{*}\left(\mathcal{O}_{\mathbb{P}^{1}}(1)\right)\right|$ be a fiber of $\varphi$, then $L^{2} F=\alpha=2,3$, respectively. Note that $\mathcal{E}=\varphi_{*}(L)$ is a rank 4 vector bundle over $\mathbb{P}^{1}, \mathcal{E}=\mathcal{O}_{\mathbb{P}^{1}}\left(a_{4}\right) \oplus \cdots \oplus \mathcal{O}_{\mathbb{P}^{1}}\left(a_{1}\right)$. We can arrange the $a_{i}$ so that $a_{4} \geq \cdots \geq a_{1}$. Then $X$ is embedded in a $\mathbb{P}^{3}$-bundle $\mathbb{P}(\mathcal{E})$ over $\mathbb{P}^{1}, \iota: X \rightarrow \mathbb{P}(\mathcal{E})$ such that $L=\iota^{*}(H)$ where $H=\mathcal{O}_{\mathbb{P}(\mathcal{E})}(1)$ is the tautological line bundle of $\mathcal{E}$ and $X \in\left|\alpha H+\rho^{*} \mathcal{O}_{\mathbb{P}^{1}}(b)\right|$, for some integer $b$, where $\rho: \mathbb{P}(\mathcal{E}) \rightarrow \mathbb{P}^{1}$ is the projection map. In what follows the notation is as above.

Proposition 4.1. Let $(X, L)$ be a 3-dimensional manifold which is either a hyperquadric fibration or a Del Pezzo fibration over $\mathbb{P}^{1}$. Let $\mathcal{E}, \alpha=2,3$, $a_{i}, i=1, \ldots, 4, b, H, \rho, \iota$, and $\varphi$ be as in 4.1. Let $d$ be the degree of $X$ and let $g$ be its sectional genus. If

i) $H^{1}(X, L)=0$,

ii) $-\alpha a_{1}-1 \leq b$,

then the Hilbert scheme of $X$ has an irreducible component, $\mathcal{H}$, which is smooth at the point representing $X$ and

$$
\operatorname{dim} \mathcal{H}= \begin{cases}d(n-4)+g(14-n)+8+3 n, & \text { if } \alpha=2 ; \\ \frac{2 d}{3}(n-14)+\frac{g}{3}(44-n)+\frac{10}{3}(10+n), & \text { if } \alpha=3 .\end{cases}
$$

Proof. Let $N$ denote the normal bundle of $X$ in $\mathbb{P}^{n}$. In order to use Proposition 2.4 we need to show that $H^{1}(X, N)=0$. Noticing that, if $\alpha=2,3$, $R^{i} \rho_{*}((1-\alpha) H)=0, i \geq 0$, the structure sequence of $X$ in $\mathbb{P}(\mathcal{E})$ tensored with $H$ gives $H^{i}(\mathbb{P}(\mathcal{E}), H)=H^{i}(X, L), i \geq 0$. On the other hand $H^{i}(\mathbb{P}(\mathcal{E}), H)=$ $H^{i}\left(\mathbb{P}^{1}, E\right)=0$, for $i \geq 2$, and thus, recalling that $H^{1}(X, L)=0$ by assumption, $H^{i}(X, L)=0, i \geq 1$.

Reasoning as in the proof of Proposition 3.1 we get that

$$
h^{i}(X, N)=h^{i+1}\left(X, T_{X}\right) \quad \text { for } \quad i \geq 1 .
$$

Hence $H^{3}(X, N)=0$ for dimension reasons. As $X \subset \mathbb{P}(\mathcal{E})$ and $X \in \mid \alpha H+$ $\rho^{*} \mathcal{O}_{\mathbb{P}^{1}}(b) \mid$, we have the following exact sequences:

$$
\begin{gathered}
0 \longrightarrow \mathcal{O}_{\mathbb{P}(\mathcal{E})}(-X) \longrightarrow \mathcal{O}_{\mathbb{P}(\mathcal{E})} \longrightarrow \mathcal{O}_{X} \longrightarrow 0, \\
0 \longrightarrow T_{X} \longrightarrow T_{\mathbb{P}(\mathcal{E}) \mid X} \longrightarrow\left(\alpha H+\rho^{*} \mathcal{O}_{\mathbb{P}^{1}}(b)\right)_{\mid X} \longrightarrow 0 .
\end{gathered}
$$


Note that $\left.\left(\alpha H+\rho^{*} \mathcal{O}_{\mathbb{P}^{1}}(b)\right)_{\mid X}=\alpha L+\varphi^{*} \mathcal{O}_{\mathbb{P}^{1}}(b)\right)=\alpha L+b F$, where $F \in$ $\left|\varphi^{*} \mathcal{O}_{\mathbb{P}^{1}}(1)\right|$ is a fiber of $\varphi$.

To compute $H^{2}\left(X, T_{X}\right)$ is enough to compute $H^{i}(X, \alpha L+b F)$ and $H^{i}\left(X, T_{\mathbb{P}(\mathcal{E}) \mid X}\right)$.

Tensoring sequence (27) with $\alpha H+\rho^{*} \mathcal{O}_{\mathbb{P}^{1}}(b)$ we get

$$
0 \longrightarrow \mathcal{O}_{\mathbb{P}(\mathcal{E})} \longrightarrow \alpha H+\rho^{*} \mathcal{O}_{\mathbb{P}^{1}}(b) \longrightarrow \alpha L+\varphi^{*} \mathcal{O}_{\mathbb{P}^{1}}(b) \longrightarrow 0 .
$$

As

$$
H^{i}\left(\mathbb{P}(\mathcal{E}), \mathcal{O}_{\mathbb{P}(\mathcal{E})}\right)=h^{i}\left(\mathbb{P}^{1}, \mathcal{O}_{\mathbb{P}^{1}}\right)=0 \text { for } i \geq 1
$$

it follows that

$$
H^{i}\left(X, \alpha L+\varphi^{*} \mathcal{O}_{\mathbb{P}^{1}}(b)\right)=H^{i}\left(\mathbb{P}(\mathcal{E}), \alpha H+\rho^{*} \mathcal{O}_{\mathbb{P}^{1}}(b)\right) \text { for } i \geq 1 .
$$

By Leray's spectral sequence

$$
H^{i}\left(\mathbb{P}(\mathcal{E}), \alpha H+\rho^{*} \mathcal{O}_{\mathbb{P}^{1}}(b)\right)=H^{i}\left(\mathbb{P}^{1}, S^{\alpha}(\mathcal{E}) \otimes \mathcal{O}_{\mathbb{P}^{1}}(b)\right),
$$

hence

$$
H^{i}\left(X, \alpha L+\varphi^{*} \mathcal{O}_{\mathbb{P}^{1}}(b)\right)=H^{i}\left(\mathbb{P}^{1}, S^{\alpha}(\mathcal{E}) \otimes \mathcal{O}_{\mathbb{P}^{1}}(b)\right)=0 \text { for } i \geq 2 .
$$

Because the $a_{i}{ }^{\prime} s$ are sorted in increasing order, the smallest degree of the line bundles of the decomposition of $S^{\alpha}(\mathcal{E}) \otimes \mathcal{O}_{\mathbb{P}^{1}}(b)$ is $\alpha a_{1}+b$. From our assumption $-\alpha a_{1}-1 \leq b$ it follows

$$
H^{1}\left(X, \alpha L+\varphi^{*} \mathcal{O}_{\mathbb{P}^{1}}(b)\right)=H^{1}\left(\mathbb{P}^{1}, S^{\alpha}(\mathcal{E}) \otimes \mathcal{O}_{\mathbb{P}^{1}}(b)\right)=0 .
$$

Tensoring sequence (27) with $T_{\mathbb{P}(\mathcal{E})}$ we get

$$
0 \rightarrow T_{\mathbb{P}(\mathcal{E})}\left(-\alpha H+\rho^{*} \mathcal{O}_{\mathbb{P}^{1}}(-b)\right) \rightarrow T_{\mathbb{P}(\mathcal{E})} \rightarrow T_{\mathbb{P}(\mathcal{E}) \mid X} \rightarrow 0 .
$$

From sequence (28) it follows that $H^{i}\left(X, T_{X}\right)=H^{i}\left(T_{\mathbb{P}(\mathcal{E}) \mid X}\right), i \geq 2$. Vanishing of these cohomology groups will follow from $H^{i+1}\left(T_{\mathbb{P}(\mathcal{E})}\left(-\alpha H+\rho^{*} \mathcal{O}_{\mathbb{P}^{1}}(-b)\right)=\right.$ $0, H^{i}\left(T_{\mathbb{P}(\mathcal{E})}\right)=0, i \geq 2$, and (30).

In order to compute such cohomology groups we consider the following exact sequences associated to $\rho: \mathbb{P}(\mathcal{E}) \rightarrow \mathbb{P}^{1}$ :

$$
\begin{gathered}
0 \rightarrow T_{\mathbb{P}(\mathcal{E}) \mid \mathbb{P}^{1}} \rightarrow T_{\mathbb{P}(\mathcal{E})} \rightarrow \rho^{*} T_{\mathbb{P} 1} \rightarrow 0, \\
0 \rightarrow \mathcal{O}_{\mathbb{P}(\mathcal{E})} \rightarrow \rho^{*} E^{*} \otimes \mathcal{O}_{\mathbb{P}(\mathcal{E})}(1) \rightarrow T_{\mathbb{P}(\mathcal{E}) \mid \mathbb{P}^{1}} \rightarrow 0 .
\end{gathered}
$$

Tensoring sequences (31) and (32) with $-\alpha H+\rho^{*} \mathcal{O}_{\mathbb{P}^{1}}(-b)$ we get, respectively,

$$
\begin{array}{r}
0 \rightarrow T_{\mathbb{P}(\mathcal{E}) \mid \mathbb{P}^{1}}\left(-\alpha H+\rho^{*} \mathcal{O}_{\mathbb{P}^{1}}(-b)\right) \rightarrow \\
T_{\mathbb{P}(\mathcal{E})}\left(-\alpha H+\rho^{*} \mathcal{O}_{\mathbb{P}^{1}}(-b)\right) \rightarrow-\alpha H+\rho^{*} \mathcal{O}_{\mathbb{P}^{1}}(2-b) \rightarrow 0, \\
0 \rightarrow-\alpha H+\rho^{*} \mathcal{O}_{\mathbb{P}^{1}}(-b) \rightarrow \rho^{*}\left(E^{*}(-b)\right) \otimes \mathcal{O}_{\mathbb{P}(\mathcal{E})}(1-\alpha) \rightarrow \\
T_{\mathbb{P}(\mathcal{E}) \mid \mathbb{P}^{1}}\left(-\alpha H+\rho^{*} \mathcal{O}_{\mathbb{P}^{1}}(-b)\right) \rightarrow 0 .
\end{array}
$$

Taking direct images of (33) via $\rho$, and noticing that, if $\alpha=2,3$, it is

$$
R^{i} \rho_{*}\left(-\alpha H+\rho^{*} \mathcal{O}_{\mathbb{P}^{1}}(-b)\right)=0, \quad \text { for } i \geq 0,
$$


it follows that

$R^{i} \rho_{*}\left(T_{\mathbb{P}(\mathcal{E}) \mid \mathbb{P}^{1}}\left(-\alpha H+\rho^{*} \mathcal{O}_{\mathbb{P}^{1}}(-b)\right)\right)=R^{i} \rho_{*}\left(T_{\mathbb{P}(\mathcal{E})}\left(-\alpha H+\rho^{*} \mathcal{O}_{\mathbb{P}^{1}}(-b)\right)\right), \quad$ for $i \geq 0$.

Similarly, taking direct images via $\rho$ of sequence (34) and noticing that $R^{i} \rho_{*}\left(-\alpha H+\rho^{*} \mathcal{O}_{\mathbb{P}^{1}}(-b)\right)=R^{i} \rho_{*}\left(\rho^{*}\left(E^{*}(-b)\right) \otimes \mathcal{O}_{\mathbb{P}(\mathcal{E})}(-2)\right)=0, \quad$ for $i \geq 0$, it follows that

$$
R^{i} \rho_{*}\left(T_{\mathbb{P}(\mathcal{E}) \mid \mathbb{P}^{1}}\left(-\alpha H+\rho^{*} \mathcal{O}_{\mathbb{P}^{1}}(-b)\right)\right)=0, \quad \text { for } i \geq 0 .
$$

Hence $H^{i}\left(\mathbb{P}(\mathcal{E}), T_{\mathbb{P}(\mathcal{E}) \mid \mathbb{P}^{1}}\left(-\alpha H+\rho^{*} \mathcal{O}_{\mathbb{P}^{1}}(-b)\right)=H^{i}\left(\mathbb{P}^{1}, \rho_{*}\left(T_{\mathbb{P}(\mathcal{E}) \mid \mathbb{P}^{1}}(-\alpha H+\right.\right.\right.$ $\left.\left.\rho^{*} \mathcal{O}_{\mathbb{P}^{1}}(-b)\right)\right)=0$, for all $i \geq 0$.

We now turn our attention to $H^{i}\left(\mathbb{P}(\mathcal{E}), T_{\mathbb{P}(\mathcal{E})}\right)$.

First notice that $\left.H^{i}\left(\mathbb{P}(\mathcal{E}), \rho^{*} \mathcal{O}_{\mathbb{P}^{1}}(2)\right)\right)=H^{i}\left(\mathbb{P}^{1}, \mathcal{O}_{\mathbb{P}^{1}}(2)\right)=0$, for $i \geq 1$. Taking direct images via $\rho$ of sequence (32) and noticing that

$$
R^{i} \rho_{*}\left(\mathcal{O}_{\mathbb{P}(\mathcal{E})}\right)=R^{i} \rho_{*}\left(\rho^{*} E^{*} \otimes \mathcal{O}_{\mathbb{P}(\mathcal{E})}(1)\right)=0, \quad \text { for } i \geq 1,
$$

it follows that

$$
0 \rightarrow \mathcal{O}_{\mathbb{P}^{1}} \rightarrow E^{*} \otimes E \rightarrow \rho_{*}\left(T_{\mathbb{P}(\mathcal{E}) \mid \mathbb{P}^{1}}\right) \rightarrow 0
$$

and

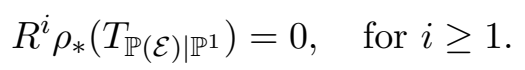

Thus by Leray's spectral sequence $H^{i}\left(\mathbb{P}(\mathcal{E}), T_{\mathbb{P}(\mathcal{E}) \mid \mathbb{P}^{1}}\right)=H^{i}\left(\mathbb{P}^{1}, \rho_{*}\left(T_{\mathbb{P}(\mathcal{E}) \mid \mathbb{P}^{1}}\right)\right)$ for $i \geq 0$. Moreover, as $H^{i}\left(\mathbb{P}^{1}, \mathcal{O}_{\mathbb{P}^{1}}\right)=0$ for $i \geq 1$ and $H^{i}\left(\mathbb{P}^{1}, E^{*} \otimes E\right)=0$

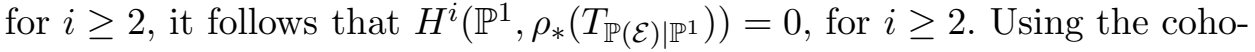
mology sequence associated to (31) we get that $H^{i}\left(\mathbb{P}(\mathcal{E}), T_{\mathbb{P}(\mathcal{E})}\right)=0, i \geq 2$. Hence $H^{i}\left(X, T_{X}\right)=0, i \geq 2$, and thus by (26) we get $H^{1}(X, N)=0, i \geq 1$.

The dimension of $\mathcal{H}$, by ([2.4), (iv)), is now obtained via HirzebruchRiemann-Roch, as in the proof of Proposition 3.1 .

One can compute easily the numerical invariants of $X$ and get:

$$
\begin{aligned}
K L^{2} & =2 g-2-2 d ; \\
K^{2} L & =\alpha d(4-\alpha)+4 g(\alpha-4)-4 \alpha+16 ; \\
c_{2} L & =\alpha d(2-\alpha)+2 g(2 \alpha-3)+2 \alpha^{2}-2 \alpha+6 ; \\
-K c_{2} & =24 ; \\
K^{3} & =2 d\left(16-24 \alpha+9 \alpha^{2}-\alpha^{3}\right)+6 g\left(16-8 \alpha+\alpha^{2}\right)-6 \alpha^{2}+48 \alpha-96 ; \\
c_{3} & =2 d\left(\alpha^{3}-3 \alpha^{2}+3 \alpha-1\right)-6 g\left(\alpha^{2}-2 \alpha+1\right)-4 \alpha^{3}+10 \alpha^{2}-6 \alpha+6 .
\end{aligned}
$$

Thus

$$
\begin{aligned}
h^{0}(N)=\chi(N)= & \frac{d}{6}\left(-\alpha^{3}+5 \alpha^{2}+4 n-n \alpha^{2}+3 n \alpha+40-38 \alpha\right)+ \\
& \frac{g}{6}\left(94-14 n-11 \alpha+3 \alpha^{2}+4 n \alpha\right)+ \\
& \frac{1}{6}\left(38 \alpha+\alpha^{3}+20 n+19 \alpha^{2}+n \alpha^{2}-3 n \alpha-112\right) .
\end{aligned}
$$


As a Corollary to Proposition 4.1 we show that all the known 3-folds which are either a hyperquadric fibration or a Del Pezzo fibration over $\mathbb{P}^{1}$ of degree $7 \leq d \leq 12$ are unobstructed and we compute the dimension of the irreducible component of the Hilbert scheme to which they belong. Note that no such varieties exist for $d \leq 6$.

Corollary 4.2. Let $(X, L)$ be as in 4.1 , with $\alpha=2$, i.e. a hyperquadric fibration over $\mathbb{P}^{1}$. Let $X$ be embedded by $|L|$ in $\mathbb{P}^{n}$ and let the numerical invariants of $X \subset \mathbb{P}^{n}$ be as in the table below. Then the Hilbert scheme of $X$ has an irreducible component, $\mathcal{H}$, which is smooth at the point representing $X$ and of dimension as in the rightmost column of the table.

\begin{tabular}{|c|c|c|c|c|c|c|}
\hline Case & $d$ & $g$ & $n$ & $b$ & Reference & $\operatorname{dim} \mathcal{H}$ \\
\hline 1 & 7 & 3 & 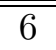 & $\overline{1}$ & [20] Theorem 4.3 and $\S 8$ & 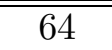 \\
\hline 2 & 8 & 3 & 7 & 0 & 20 Theorem 4.3 & 74 \\
\hline 3 & 9 & 3 & 8 & -1 & 14] Theorem 3.3 & 86 \\
\hline 4 & 9 & 4 & 7 & 1 & 14 Theorem 3.3 & 84 \\
\hline 5 & 10 & 3 & 9 & -2 & 15] Prop. 3.4 & 100 \\
\hline 6 & 10 & 4 & 8 & 0 & 15] Prop. 3.4 & 96 \\
\hline 7 & 10 & 5 & 7 & 2 & 15. Prop. 7.1 & 94 \\
\hline 8 & 11 & 3 & 10 & -3 & $\begin{array}{ll}6 & \text { Prop. 4.2.2 } \\
\end{array}$ & 116 \\
\hline 9 & 11 & 4 & 9 & -1 & [6] Prop. 4.2.3, Remark 4.2.5 & 110 \\
\hline 10 & 11 & 5 & 8 & 1 & \begin{tabular}{|l|l|}
6 & Prop. 5.2.1 \\
\end{tabular} & 106 \\
\hline 11 & 11 & 6 & 7 & 3 & $\begin{array}{lll}6] & \text { Prop. 5.2.1 } \\
\end{array}$ & 104 \\
\hline
\end{tabular}

Proof. We will show that the hypothesis of Proposition 4.1 are satisfied for all cases in the above table. The structure sequences of a general surface section $S$ and curve section $C$ tensored with $L$ and $L_{\left.\right|_{S}}$ respectively, give

$$
h^{0}(L)-h^{1}(L)=2+\chi\left(\mathcal{O}_{S}\right)+d-g .
$$

On the other hand, $h^{0}(L)=n+1$ and thus

$$
h^{1}(L)=n-1-\chi\left(\mathcal{O}_{S}\right)-d+g .
$$

Noticing that $\chi\left(\mathcal{O}_{S}\right)=1$, being $S$ a conic bundle over $\mathbb{P}^{1}$, a simple check gives $h^{1}(L)=0$ for all cases in the above table.

As in the proof of [17, Lemma 3.19, in all cases above, but 7 and 11, as $b \leq 1$, it follows that $a_{1} \geq 0$. A simple numerical check, independent of the value of $a_{1} \geq 0$ shows that hypothesis ii) in Proposition 4.1 is verified in cases 1 through 4, 6, 9 and 10 .

In cases 5 and 8 , 13. Theorem 2.0 gives $a_{1}=1$ and thus hypothesis ii) in Proposition 4.1 is satisfied.

Because $H^{1}\left(\mathbb{P}^{1}, \mathcal{E}\right)=H^{1}(X, L)=0$, it follows that $a_{1} \geq-1$. Therefore hypothesis ii) in Proposition 4.1 is verified in cases 7 and 11 .

By Proposition 4.1 there exists an irreducible component $\mathcal{H}$ of the Hilbert scheme of $X \subset \mathbb{P}^{n}$ whose dimension can now be easily computed. 
Corollary 4.3. Let $(X, L)$ be as in 4.1, with $\alpha=3$, i.e. a Del Pezzo fibration over $\mathbb{P}^{1}$ with fiber degree 3 . Let $X$ be embedded by $|L|$ in $\mathbb{P}^{n}$ and let the numerical invariants of $X \subset \mathbb{P}^{n}$ be as in the table below, where $S \in|L|$ is a general surface section. Then the Hilbert scheme of $X \subset \mathbb{P}^{n}$ has an irreducible component, $\mathcal{H}$, which is smooth at the point representing $X$ and of dimension as in the rightmost column of the table.

\begin{tabular}{|c|c|c|c|c|c|c|}
\hline$d$ & $g$ & $n$ & $p_{g}(S)$ & $b$ & Reference & $\operatorname{dim} \mathcal{H}$ \\
\hline \hline 9 & 7 & 6 & 2 & 0 & {$[14]$ Prop. 2.5, } & 94 \\
\hline 10 & 9 & 6 & 3 & 1 & {$[15]$ Theorem 4.2, } & 114 \\
\hline 11 & 8 & 7 & 2 & -1 & {$[6]$ Remark 5.4.7 } & 104 \\
\hline
\end{tabular}

Proof. We will show that the hypothesis of Proposition 4.1] are satisfied for all cases in the above table.

Noticing that formula (37) holds true in these cases and that $q(S)=0$, a simple check gives $h^{1}(L)=0$.

As in the proof of [17], Lemma 3.19, because in all cases above it is $b \leq 2$, it follows that $a_{1} \geq 0$. A simple numerical check now shows that hypothesis ii) in Proposition 4.1 is verified in all the cases of the above table.

By Proposition 4.1 there exists an irreducible component $\mathcal{H}$ of the Hilbert scheme of $X \subset \mathbb{P}^{n}$ whose dimension can now be easily computed.

\section{Good Determinantal Varieties}

In 23] results are obtained on the unobstructedness of good determinantal subschemes, as an application of the authors' remarkable G-liaison theory. In particular, all good determinantal subschemes of codimension 3 were shown to be unobstructed and the dimension of their locus inside the Hilbert scheme was computed. Some of the varieties considered in previous sections of this work have explicit constructions, available in the literature, which easily show that they are examples of good determinantal schemes. This section addresses the relationship between our work and [23].

For the convenience of the reader we begin the section by recalling the definition of good determinantal subscheme and the basic notation utilized in 23 .

Definition 5.1 (cf.Definition 3.1 in [23]). Let $A$ be a homogenous matrix, i.e $A=\left[f_{i, j}\right]$ where $f_{i, j} \in \mathbb{C}\left[x_{0}, \ldots x_{n+c}\right]$ is a homogeneous polynomial of degree $d_{i j}$. Let $I(A)$ denote the ideal of maximal minors of $A$. A codimension $c$ scheme, $X$, in $\mathbb{P}^{n+c}$ will be called standard determinantal scheme if $I_{X}=$ $I(A)$ for some homogeneous $t \times(t+c-1)$ matrix, $A$. $X$ will be called a good determinantal scheme if additionally, $A$ contains a $(t-1) \times(t+c-1)$ submatrix (allowing a change of basis if necessary) whose ideal of maximal minors defines a subscheme of codimension $c+1$. If $u: F \rightarrow E$ is a vector bundle homomorphism, over $\mathbb{P}^{n+c}$, we define $I(u)=I(A)$ for any homogeneous matrix representing $u$. 
Note that the matrix $A$ defines a morphism of locally free sheaves

$$
u: \oplus_{i=1}^{t} \mathcal{O}_{\mathbb{P}^{n+c}}\left(b_{i}\right) \rightarrow \oplus_{j=0}^{t+c-2} \mathcal{O}_{\mathbb{P}^{n+c}}\left(a_{j}\right)
$$

where $d_{i j}=a_{j}-b_{i}, b_{1} \geq \cdots \geq b_{t}$ and $a_{0} \geq \cdots \geq a_{t+c-2}$.

The locus of good determinantal subschemes $X$ in $\mathbb{P}^{n+c}$ of codimension $c$ defined by a matrix $A$ as above, following 23 , is denoted by $W(\underline{b}, \underline{a})$, where $W(\underline{b}, \underline{a})$ stands for $W\left(b_{1}, \ldots, b_{t} ; a_{0}, \ldots, a_{t+c-2}\right), W(\underline{b}, \underline{a}) \subset \operatorname{Hilb}^{p(t)}\left(\mathbb{P}^{n+c}\right)$, where $p(t)$ is the Hilbert polynomial of $X$.

The following examples are explicit constructions of some of the varieties which appear in the Table in Corollaries 3.3 4.3 and 4.2. respectively.

Example 5.2. Let $F=\mathcal{O}_{\mathbb{P}^{6}}^{\oplus 3}$ and $E=\mathcal{O}_{\mathbb{P}^{6}}(1)^{\oplus 5}$ be vector bundles on $\mathbb{P}^{6}$ and let $u: \mathcal{O}_{\mathbb{P} 6}^{\oplus 3} \rightarrow \mathcal{O}_{\mathbb{P} 6}(1)^{\oplus 5}$ be a generic vector bundle homomorphism. Let $I(u)=I(A)$, where $A$ is a homogeneous matrix representing $u$ and where $I(A)$ denotes the ideal of maximal minors of $A$. Let $X_{1}$ be the determinantal variety whose ideal $I_{X_{1}}=I(u)$. It can be easily seen that any such $X_{1}$ is a smooth threefold in $\mathbb{P}^{6}$ with $\operatorname{deg} X_{1}=10, g\left(X_{1}\right)=6$, Hilbert polynomial $p_{1}(t)=\frac{5}{3} t^{3}+4 t^{2}+\frac{10}{3} t+1$ and that it has the structure of a scroll over $\mathbb{P}^{2}$, see (15], Prop. 5.2). It appears in row 4 of the Table in Corollary 3.3

It is straightforward from Definition 5.1 to see that this variety is a good determinantal subscheme of $\mathbb{P}^{6}$.

Example 5.3. Let $E_{2}=\mathcal{O}_{\mathbb{P}^{6}}(1)^{\oplus 3} \oplus \mathcal{O}_{\mathbb{P}^{6}}(3), E_{3}=\mathcal{O}_{\mathbb{P}^{6}}(1)^{\oplus 3} \oplus \mathcal{O}_{\mathbb{P}^{6}}(2)$ and $F=\mathcal{O}_{\mathbb{P} 6}^{\oplus 2}$ be vector bundles over $\mathbb{P}^{6}$. Let $u_{i}: F \rightarrow E_{i}$, for $i=2,3$, be generic vector bundle homomorphisms and let $X_{i}$ be the determinantal varieties whose ideals $I_{X_{i}}=I\left(u_{i}\right)$. It can be easily seen that $\operatorname{deg} X_{2}=10$ and $g\left(X_{2}\right)=9$ while $\operatorname{deg} X_{3}=7$ and $g\left(X_{3}\right)=3$.

Any such $X_{2}$ is a smooth threefold, known to be a Del Pezzo fibration of fiber degree 3 over $\mathbb{P}^{1}$, see ([15], Remark 4.3), with Hilbert polynomial $p_{2}(t)=\frac{5}{3} t^{3}+t^{2}+\frac{10}{3} t+1$. It appears in row 2 of the Table in Corollary 4.3

Any such $X_{3}$ is a smooth threefold, known to be a quadric fibration over $\mathbb{P}^{1}$, with Hilbert polynomial $p_{3}(t)=\frac{7}{6} t^{3}+\frac{5}{2} t^{2}+\frac{7}{3} t+1$. It appears in the first row of the Table in Corollary 4.2.

In both cases it is straightforward from Definition 5.1 to see that these varieties are good determinantal subschemes of $\mathbb{P}^{6}$.

Proposition 5.4. Let $X \subset \mathbb{P}^{6}$ be a threefold of degree $d$, genus g, with Hilbert polynomial $p(t)$, as in one of the cases in the following table 


\begin{tabular}{|c|c|c|c|c|}
\hline$d$ & $g$ & $p(t)$ & Geometric Structure & Reference \\
\hline \hline 7 & 3 & $\frac{7}{6} t^{3}+\frac{5}{2} t^{2}+\frac{7}{3} t+1$ & $\begin{array}{c}\text { Hyperquadric fibration } \\
\text { over } \mathbb{P}^{1}\end{array}$ & $\begin{array}{c}\text { Corollary 4.2. } \\
\text { 20] Theorem 4.3 and } \S 8\end{array}$ \\
\hline 10 & 6 & $\frac{5}{3} t^{3}+4 t^{2}+\frac{10}{3} t+1$ & Scroll over $\mathbb{P}^{2}$ & $\begin{array}{c}\text { Corollary [3.3, } \\
\text { 15] Remark 5.3, }\end{array}$ \\
\hline 10 & 9 & $\frac{5}{3} t^{3}+t^{2}+\frac{10}{3} t+1$ & $\begin{array}{c}\text { DelPezzo fibration } \\
\text { over } \mathbb{P}^{1} \text { of fiber degree 3 }\end{array}$ & $\begin{array}{c}\text { Corollary [.3. } \\
\text { 15] Theorem 4.2 }\end{array}$ \\
\hline
\end{tabular}

Then:

i) $\operatorname{Hilb}^{p(t)}\left(\mathbb{P}^{6}\right)$ has an irreducible component $\mathcal{H}$ of dimension, respectively, $\operatorname{dim} \mathcal{H}=64,72,114$ and $X$ corresponds to a smooth point of $\mathcal{H}$;

ii) There exists an open subset $U \in \mathcal{H}$ whose points correspond to good determinantal subschemes given by morphisms of vector bundles

$$
u: \oplus_{i=1}^{t} \mathcal{O}_{\mathbb{P}^{6}}\left(b_{i}\right) \rightarrow \oplus_{j=0}^{t+1} \mathcal{O}_{\mathbb{P}^{6}}\left(a_{j}\right)
$$

with $b_{1} \geq \cdots \geq b_{t}$ and $a_{0} \geq \cdots \geq a_{t+1}$

iii) Let $W(\underline{b}, \underline{a}) \subset \operatorname{Hilb}^{p(t)}\left(\mathbb{P}^{6}\right)$ be the locus of good determinantal subschemes where $\underline{a}=\left\{a_{0}, \ldots, a_{t+1}\right\}$ and $\underline{b}=\left\{b_{0}, \ldots, b_{t}\right\}$ are as in ii). Then $\mathcal{H}$ is the closure of $W(\underline{b}, \underline{a})$ in $H_{i l b} b^{(t)}\left(\mathbb{P}^{6}\right)$

Proof. Statement i) follows from Corollaries 3.3. 4.2 and 4.3 . Statement ii) follows from Examples 5.2 and 5.3. Let $X_{i}, i=1,2,3$ be as in Examples 5.2 and 5.3. One can easily check that all of these varieties satisfy the hypotheses of [23, Corollary 10.15] and thus they are unobstructed. Let $W_{i}$ denote the locus of good determinantal subschemes with Hilbert polynomial $p_{i}(t)$ to which the $X_{i}$ belong. The same corollary gives, respectively, $\operatorname{dim} W_{i}=$ $72,64,114$ for $i=1,2,3$.

Hence $\mathcal{H}$ and the locus $W_{i}$ of good determinantal subschemes in $\mathbb{P}^{6}$ have the same dimension. As each $X_{i}$ is a smooth point of $\mathcal{H}$, it must be $\mathcal{H}=\bar{W}$, where $\bar{W}$ denotes the closure of $W$ in $\operatorname{Hilb}^{p(t)}\left(\mathbb{P}^{6}\right)$. Note that this is not true in general, see [23, Example 10.5 (4)].

\section{REFERENCES}

[1] H. Abo, W. Decker, and N. Sasakura. An elliptic conic bundle in $\mathbb{P}^{4}$ arising from a stable rank-3 vector bundle. Math. Z., 229(4):725-748, 1997.

[2] M. Beltrametti, M. Schneider, and A. J. Sommese. Threefolds of degree 9 and 10 in $\mathbf{P}^{5}$. Math. Ann., (288):613-644, 1990.

[3] M. Beltrametti, M. Schneider, and A. J. Sommese. Threefolds of degree 11 in $\mathbf{P}^{5}$. In Complex Projective Geometry, Bergen - Trieste, number 179 in London Math. Soc. Lecture Notes, pages 59-80, 1992. 
[4] M. Beltrametti and A. J. Sommese. The Adjunction Theory of Complex Projective Varieties, volume 16 of Expositions in Mathematics. De Gruyter, 1995.

[5] M. Bertolini. Threefolds in $\mathbb{P}^{6}$ of degree twelve. Preprint, 2004.

[6] G. M. Besana and A. Biancofiore. Degree eleven manifolds of dimension greater or equal to three. Forum Mathematicum, to appear, 2004.

[7] A. Biancofiore and M. L. Fania. On the structure of linked threefolds. Rev. Mat. Complut., (1):17-44, 2001.

[8] R. Braun, G. Ottaviani, M. Schneider, and F.-O. Schreyer. Boundedness for nongeneral-type 3-folds in $\mathbf{P}_{5}$. In Complex analysis and geometry, Univ. Ser. Math., pages 311-338. Plenum, New York, 1993.

[9] M.-C. Chang. The number of components of Hilbert schemes. Internat. J. Math., $7(3): 301-306,1996$.

[10] M.-C. Chang. Inequidimensionality of Hilbert schemes. Proc. Amer. Math. Soc., 125(9):2521-2526, 1997.

[11] H. D'Souza. Threefolds whose hyperplane sections are elliptic surfaces. Pacific J. Math., 134(1):57-78, 1988.

[12] G. Ellingsrud. Sur le schéma de Hilbert des variétés de codimension 2 dans $\mathbb{P}^{e}$ a cône de Cohen-Macaulay. Ann. scient. Éc. Norm. Sup. $4^{e}$ serie, (8):423-432, 1975.

[13] M. L. Fania and E. L. Livorni. Polarized manifolds of dimension $\geq 3, \Delta$-genus three, $\operatorname{dim} \operatorname{Bs}|L| \leq 0$ and degree $\geq 2 \Delta-1$. Saitama Math. Journal, (11), 1993.

[14] M. L. Fania and E. L. Livorni. Degree nine manifolds of dimension $\geq 3$. Math. Nachr., (169):117-134, 1994.

[15] M. L. Fania and E. L. Livorni. Degree ten manifolds of dimension $n$ greater than or equal to 3. Math. Nachr., (188):79-108, 1997.

[16] M. L. Fania and E. Mezzetti. On the Hilbert scheme of Palatini threefolds. Advances in Geometry, (2):371-389, 2002.

[17] T. Fujita. Classification of polarized manifolds of sectional genus two. In Algebraic Geometry and Commutative Algebra in honor of Masayoshi Nagata, pages 73-98. Kinokuniya, 1987.

[18] A. Grothendieck. Techniques de construction et théorèmes d'existence en géométrie algébrique. IV: les schémas de Hilbert. Number 221 in Seminaire Bourbaki. 1960.

[19] R. Hartshorne. Algebraic Geometry. Number 52 in GTM. Springer Verlag, New York - Heidelberg - Berlin, 1977.

[20] P. Ionescu. Embedded projective varieties of small invariants. In Proceedings of the Week of Algebraic Geometry Bucharest 1982, number 1056 in Lecture Notes in Mathematics, pages 142-186. Springer Verlag, 1984.

[21] P. Ionescu. Generalized adjunction and applications. Math. Proc. Camb. Phil. Soc., (99):457-472, 1986.

[22] P. Ionescu. Embedded projective varieties of small invariants III. In Algebraic Geometry L'Aquila 1988, number 1417 in Lecture Notes in Mathematics, pages 138-154. Springer Verlag, 1990.

[23] J. O. Kleppe, J. C. Migliore, R. Miró-Roig, U. Nagel, and C. Peterson. Gorenstein liaison, complete intersection liaison invariants and unobstructedness. Mem. Amer. Math. Soc., 154(732):viii+116, 2001.

[24] J. O. Kleppe and R. M. Miró-Roig. The dimension of the Hilbert scheme of Gorenstein codimension 3 subschemes. J. Pure Appl. Algebra, (127):73-82, 1998.

[25] C. Okonek. Über 2-codimensionale Untermannigfaltigkeiten vom Grad $7 \mathrm{im} \mathbf{P}^{4}$ und $\mathbf{P}^{5}$. Math. Z., (187):209-219, 1984.

[26] C. Okonek. Flächen vom Grad 8 im $\mathbf{P}^{4}$. Math. Z., (191):207-223, 1986.

[27] C. Okonek. Notes on varieties of codimension 3 in $\mathbf{P}^{n}$. Manuscripta Math., 84:421442, 1994. 
[28] C. Okonek, M. Schneider, and H. Spindler. Vector Bundles on Complex Projective Spaces. Number 3 in Progress in Mathematics. Birkhäuser, Boston - Basel - Stuttgart, 1980.

[29] G. Ottaviani. On 3-folds in $\mathbb{P}^{5}$ which are scrolls. Annali della Scuola Normale di Pisa Scienze Fisiche e Matematiche, IV - XIX(3):451-471, 1992.

[30] A. J. Sommese. On the minimality of hyperplane sections of projective threefolds. $J$. Reine Angew. Math., (329):16-41, 1981.

[31] H. Swinnerton Dyer. An enumeration of all varieties of degree 4. American Journal of Math, (95):403-418, 1973.

[32] XXX. Correspondence. American Journal of Mathematics, (79):951-952, 1957. Now in A. Weil, Oeuvres Sc. II, 555-556.

Gian Mario Besana, C.T.I. DePaul University, 243 S. Wabash, Chicago IL 60604, USA

E-mail address: gbesana@cs.depaul.edu

Maria Lucia Fania, Dipartimento di Matematica, Università degli Studi di L'Aquila, Via Vetoio Loc. Coppito, 67100 L'Aquila, Italy

E-mail address: fania@univaq.it 